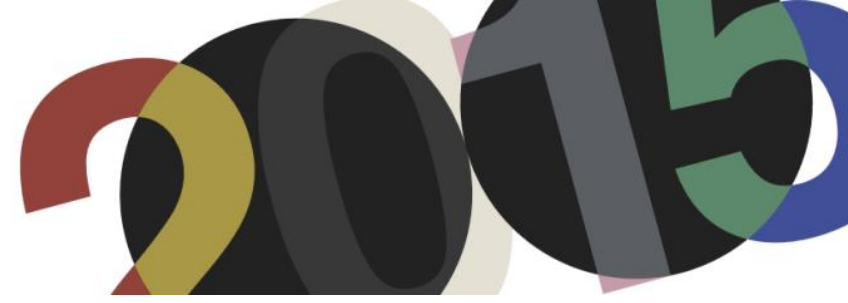

DOI: http://dx.doi.org/10.4995/LC2015.2015.682

\title{
Le Corbusier y la autonomía de la arquitectura
}

\author{
A. Rubio Garrido
}

Escuela Técnica Superior de Arquitectura de Valencia

\begin{abstract}
Resumen: Tres han sido los intentos de vincular la obra de Le Corbusier con la "autonomía de la arquitectura".
El primero, aquel formulado por Emil Kaufmann en 1933, reposa en una analogía ética conducida por medio de ciertos mecanismos compositivos. El segundo lo alentó Johnson durante la posguerra e identifica en Le Corbusier un sistema de composición autorreferencial pretendidamente ajeno a todo condicionante exterior que enfatiza el purismo de sus operaciones formales. El tercero fue conducido por un grupo de autores englobados en el "autonomy project" que vio en su obra la posibilidad de entender la arquitectura simultáneamente como hecho social y producto formal.

Cada intento ensalzó ciertas características de Le Corbusier y obvió otras en la medida en que se adscribieron a las diversas, y por momentos divergentes, interpretaciones de la autonomía de la arquitectura.

En este texto defenderé que la aparente multiplicidad de las interpretaciones de la obra de Le Corbusier que derivan de estos intentos puede superarse atendiendo al sentido filosófico originario de "autonomía" y su incorporación al arte. A la postre, remiten a una paradoja difícilmente superable y que puede identificarse como consustancial a la modernidad arquitectónica: la dialéctica entre la forma y la función.
\end{abstract}

Abstract: There have been three attempts to link Le Corbusier's work with the "autonomy of architecture".

The first one, the one conducted by Emil Kaufmann in 1933, lies in an ethical analogy through some compositional mechanism. Johnson encouraged the second one during the postwar. He identified in Le Corbusier a system of self-referential composition, supposedly alien to all outside constraint, which emphasizes the purity of its formal operations. The third was led by a group of authors, members of the "autonomy project". They saw in his work the possibility of understanding architecture simultaneously as a social fact and formal product.

Each attempt praised certain features of Le Corbusier and others were disregarded, following the pattern of controversial interpretations of the autonomy of architecture.

In this paper I will argue that this apparent multiplicity of interpretations of Le Corbusier's work can be overcome taking the original philosophical sense of "autonomy" and its incorporation into art. In the end, they refer to an insurmountable paradox, which can be identified as integral to modern architecture: the dialectic between form and function.

Palabras clave: Le Corbusier, autonomía de la arquitectura, compromiso, formalismo, dialéctica. Keywords: Le Corbusier, autonomy of architecture, commitment, formalism, dialectics.

\section{Introducción}

A lo largo del siglo XX, tres han sido los intentos de vincular la obra de Le Corbusier y la "autonomía de la arquitectura".

El primero reposa en una analogía ética. Apenas tres meses después del incendio del Reichstag, Kaufmann propone en su publicación Von Ledoux bis Le Corbusier. Ursprung und Entwicklung der autonomen Architektur una continuidad entre los valores democráticos y republicanos de la Revolución Francesa con la arquitectura de Le Corbusier, "el más autoconsciente protagonista" de la nueva arquitectura, "den Führer des jungen Frankreich". Respaldado por su teoría del pavillonsystem, Kaufmann encuentra en Ledoux el precedente de una arquitectura paradigmáticamente reflejada en Le Corbusier. La lucha por la autonomía se entiende en este primer 
sentido como la lucha de la autodeterminación contra el dictado de la necesidad externa, la razón contra la contingencia, lo sintético contra lo orgánico, lo puro contra lo corrupto.

El segundo está marcado por el giro que impone Kaufmann a su producción teórica a partir de su exilio en Estados Unidos de América. Durante su debut en 1942 en la American Society of Architectural Historians, Kaufmann obvia toda referencia al republicanismo revolucionario en su producción teórica (y los vínculos establecidos con Kant o Rousseau) e invierte la prioridad entre la forma y sistema en beneficio de lo que llamará el "new individualism". Toda mención al carácter emancipatorio y su analogía entre Ledoux y Le Corbusier desaparece, dando lugar a un tipo de formalismo abiertamente deudor del legado de Durand y Dubut que será explotado con profusión por Johnson, entre otros. Este constituye la segunda versión del vínculo entre Le Corbusier y la autonomía de la arquitectura: un sistema de composición autorreferencial, pretendidamente ajeno a todo condicionante exterior, que enfatiza el purismo de sus operaciones formales.

En tercer lugar, diversos autores reunidos en torno a lo que se llamó el "autonomy project" intentaron en los años setenta rehabilitar la "autonomía de la arquitectura" desde la premisa de la necesidad de incorporar una orientación social. Esta motivación inicial llevó, a la postre, a una combinación de los dos postulados anteriores, de suerte que vieron en la obra de Le Corbusier la posibilidad de entender la arquitectura simultáneamente como hecho social y producto formal.

Tras una breve introducción del concepto de autonomía desde su originaria concepción filosófica y los desafíos concretos que esta implica para la arquitectura, presentaré en los tres apartados siguientes una genealogía de las interpretaciones que han caracterizado la recepción de la "autonomía de la arquitectura" a través de la obra de Le Corbusier: el compromiso ético defendido por Kaufmann, el formalismo de Johnson y, finalmente, una suerte de relativismo condensado en el "autonomy project". Mostraré cómo, a la postre, todas remiten a una paradoja difícilmente superable y que no es otra que aquella a la que nos aboca necesariamente la asunción de la modernidad en la arquitectura: la dialéctica entre la forma y la función.

\section{La "autonomía de la arquitectura"}

como la arquitectura no es sólo autónoma, sino que al mismo tiempo es funcional, no puede negar simplemente a los seres humanos tal como son, aunque tiene que hacer esto en tanto que autónoma.

Adorno

En un sentido amplio, se entiende por "autonomía" la no dependencia de algo o alguien a la hora de determinar sus propias leyes. Así, se dice de alguien que es autónomo en la medida en que se vale por sí mismo; referido a un estado, autónomo es aquel que tiene la potestad de regirse mediante normas y órganos propios; y así en adelante. En sintonía con esta acepción, en la tradición filosófica cabe distinguir, al menos, dos amplios sentidos en su uso. En un primer sentido, que podría ser llamado ontológico, la autonomía responde a la propiedad de ciertas esferas de la realidad de regirse por leyes propias, esto es, distintas de las de otras esferas de la realidad. Aquello que distinguiría esta propiedad de la independencia sería el hecho de que, junto con estas leyes que remiten a esta esfera (aunque no forzosamente de forma exclusiva), pueden coexistir otras consideradas de rango más fundamental que, a su vez, pertenecen a otras esferas. El segundo sentido en el que se emplea la autonomía pertenece al ámbito ético. Procede originariamente del área política -y a partir del siglo

\footnotetext{
1 Adorno, Theodor W.: Teoría estética. Obra completa $n^{\circ} 7$. Rolf Tiedemann (Ed.). Jorge Navarro Pérez (Trad.). Madrid: Akal, 2004. p. 342.
} 
XVII también jurídica- y no será hasta principios de la Edad Moderna cuando puede encontrarse mención explícita en referencias aisladas de diferentes autores. En líneas generales se habla de una ley moral autónoma cuando "tiene en sí misma su fundamento y la razón propia de su legalidad".2

En cualquier caso, es a partir de Kant cuando la autonomía ocupa una posición central como concepto filosófico ${ }^{3}$ y cobra un sentido enfático. En efecto, Kant sitúa el concepto de "autonomía" en el centro de su filosofía práctica. Afirma que la razón humana es una fuente autónoma de principios de conducta, a cubierto de los halagos de inclinación sensual, tanto en sus determinaciones de valor como en sus decisiones de actuar. Así, la autonomía humana es el valor más alto y la condición de posibilidad de todos los otros valores y la "autonomía de la voluntad" [Autonomie des Willens] es entendida como supremo principio de la moralidad. ${ }^{4}$ Sería este un primer sentido de autonomía en la obra de Kant: uno indisolublemente ligado a la filosofía práctica.

Adicionalmente, puede rescatarse un segundo sentido -quizás más laxo- de autonomía en Kant. Pese a que sólo pueda aplicarse el sentido estricto de "autonomía" al dominio de la razón práctica, Kant sí defiende la autonomía del juicio del gusto como facultad del alma respecto del entendimiento y de la razón. Así, la estética sería autónoma respecto del conocimiento (no aporta conocimiento) y de la moral (es desinteresada). Es más, la producción en este ámbito, es decir, la obra de arte y la movilización de una técnica propia, tiene unos fundamentos y aspira a cierta objetividad que no son ni la del entendimiento (cuyos principios son conceptos determinantes) ${ }^{5}$ ni de la razón (cuyo principio es un ideal de perfección moral fundamentado en el imperativo moral). ${ }^{6}$ En primer lugar, la producción artística se basa en el principio de la "finalidad sin fin" mediado por la figura del genio, quien se da a sí mismo las reglas para la producción artística de tal forma que se pueda producir como si lo producido fuera obra de la naturaleza. ${ }^{7} \mathrm{Y}$, en segundo lugar, el juicio del gusto tiene por fundamento un sentimiento subjetivo desinteresado y aspira a una cierta universalidad diferente a la de las leyes naturales y la de los imperativos morales. Se fundamenta antes bien en el consenso que cabría esperar en un colectivo de gusto educado, lo que podría provocativamente llamarse una "objetividad subjetiva". ${ }^{8}$ Luego, en un sentido no tan estricto como el moral, sí puede hablarse en Kant de un tipo de autonomía del arte en la medida en que no es ni conocimiento ni moral. Esto evidenciaría una insalvable diferencia entre las producciones humanas que, como es el caso de la artesanía, no son propiamente autónomas, puesto que están orientadas a un fin. Pero puede incluso ampliarse a otras producciones como la gastronomía que, aún siendo independientes de la ciencia o de la moral, no por ello son autónomas en el sentido en que lo es el arte.

En definitiva, son igualmente identificables en Kant los dos sentidos de autonomía filosófica reseñables en el conjunto de la filosofía moderna, de suerte que puede achacársele tanto la responsabilidad de haber otorgado a la autonomía un papel central en el desarrollo del arte occidental como de posibilitar su intrínseca ambigüedad. De hecho, esta indeterminación dará lugar en la tradición estética a dos corrientes no siempre claramente diferenciables que atravesarán la exposición de este trabajo. En el primer caso, la autonomía es interpretada como libertad e independencia de cualquier manifestación artística respecto de restricciones socioculturales e

\footnotetext{
2 Véase la entrada "autonomía” en Ferrater Mora, José: Diccionario de filosofía. Buenos Aires: Editorial Sudamericana, 1964. Tomo I, p. 161.

${ }^{3}$ Ritter, Joachim (Ed.): Historisches Wörterbuch der Philosophie. Basilea: Schwabe, 2007. pp. 702-703.

${ }^{4}$ En su Fundamentación de la metafísica de las costumbres de 1785, la autonomía de la voluntad se define como "el estado por el cual ésta [la voluntad] es una ley para sí misma, independientemente de cómo están constituidos los objetos del querer”. Véase Kant, Immanuel: Fundamentación de la metafísica de las costumbres. Madrid: Espasa Calpe, 1990. p. 120.

${ }^{5}$ Véase Kant, Immanuel: Crítica del Juicio. Manuel García Morente (trad.), Madrid: Tecnos, 2007. p. 143

${ }^{6}$ Ibíd., pp. 121-122.

${ }^{7}$ Ibíd., pp. 232-234.

${ }^{8}$ Ibíd., p. 129.
} 
institucionales (estado, sociedad, Iglesia, partidos...). Es característica de la lucha por la liberación del arte y los artistas en la Edad Moderna e Ilustración respecto de todo ámbito que no sea específicamente artístico, que no tenga propiamente fines artísticos. Atiende, pues, a la exigencia de la delimitación de las esferas de la cultura. En el segundo caso, la autonomía en el arte adquiere un impulso ético. Constituiría el requisito necesario para poder establecer una nueva utopía ajena a la sociedad doliente: sólo constituyéndose el arte como autónomo, puede proponer o proporcionar alternativas a las dinámicas socioculturales que se entienden como perniciosas. Este presupuesto es propio del pensamiento revolucionario que centra todos sus esfuerzos en la transformación de las condiciones materiales de la sociedad. Aunque su desarrollo más maduro llegará con las estéticas marxistas, ya en Schiller puede identificarse este compromiso con la sociedad desde el arte.

Si esto es así en el caso del arte en general, hay que reconocer que la arquitectura establece un particular desafío a las teorías estéticas. Por sus cualidades propias, es indudable que la arquitectura constituye un caso singular entre las artes. Y es que, en tanto que arte particular, la arquitectura arrastra una serie de determinaciones internas que parecen imponer limitaciones a su autonomía, tanto en el plano formal (por su orientación funcional y por ser un arte político), como en el plano disciplinario (en tanto que arte de conjunto y arte público). ${ }^{9}$ Luego, desde la consolidación del discurso autónomo en la cultura occidental y atendiendo a las dos corrientes antes mencionadas, la arquitectura ha de enfrentarse por fuerza a una conflictiva decisión: o bien asumirse como parte indisoluble de la sociedad que le da lugar, corriendo el riesgo de mimetizarse con ella y perder, así, el carácter crítico que toda aproximación moderna exige; o bien, asumirse como autónoma, desprenderse de la sociedad y autolegitimarse. Ambos polos entrañan altos riesgos. Si confundirse con la sociedad, es decir, desprenderse de toda instancia a la que recurrir frente a los medios de presión del momento (culturales, económicos, institucionales) vulneraría el principio básico de mejorar la sociedad a la que sirve, erigirse como autónoma y desde ahí imponer una soberanía estética en "el mundo de la vida" instauraría un tipo de autoritarismo contrario a su naturaleza social. ${ }^{10}$

Pese a esta ineludible conflictividad, a principios del siglo XX se constituyó una corriente de práctica y teoría arquitectónica que tenía como eje vertebrador un decidido empeño por fundar algo así como una "arquitectura autónoma”. Fue sin lugar a dudas Emil Kaufmann el responsable de este insólito auge. Heredero de la célebre Escuela de Viena de Historia del Arte, mantuvo en sus trabajos el característico énfasis de esta tradición académica en el desarrollo de aproximaciones políticas y sociales a la teoría del arte. En este ambiente, entre profesores como Max Dvorak y Hans Semper y con el legado aún candente de las lecciones impartidas por Alois Riegl ${ }^{11}$ y Franz Wickhoff, cabe situar el periodo de formación de Kaufmann. La novedosa orientación de la historia de la arquitectura desarrollada por estos maestros -que fijaron su atención en periodos de transición o

\footnotetext{
${ }^{9}$ Estoy adaptando libremente lo que entiende Roger Scruton por "distinguishing features of architecture": literalmente, su "utility or function", su "highly localized quality", su "character as a public object" y su "continuity with the decorative arts, and the corresponding multiplicity of its aims". Véase Scruton, Roger: The Aesthetics of Architecture. Londres: Methuen \& Co., 1979. pp. 1-20.

${ }^{10}$ Sobre esto he tenido ocasión de profundizar en Rubio Garrido, Alberto: "El desafío de la expresión. Una aproximación desde la polémica de la autonomía de la arquitectura”. En Campillo, Antonio y Manzanero, Delia (Coord.): Los retos de la Filosofía en el siglo XXI. Valencia: PUV, 2015. Vol. III, pp. 49-61.

${ }^{11}$ En Riegl puede rastrearse un primer compromiso con la autonomía en la arquitectura, aunque no se mencione explícitamente. En su obra defiende que el valor del arte ha de medirse en relación a las técnicas y a los procedimientos aplicados, a la inmanencia del trabajo que contiene él mismo, a la transformación que el tiempo hace sufrir a los modelos espaciales. El arte, desde esta perspectiva, es la expresión del Wollen característico de una época. Las técnicas de realización de la forma (que hacen el lenguaje) son convencionales y por lo tanto equívocas, sujetas a transformación. El Kunstwollen se entiende como principio que informa la obra en el plano formal en el dominio de la pura visibilidad donde, aislados, entran en juego "el contorno y el color, en el plano o el espacio".
} 
decadencia obviados por la imposición de los grandes estilos- posibilitó la reivindicación de los momentos de quiebra, de ruptura, en la historia de la arquitectura como periodos merecedores de ser estudiados sin supeditarse a la evolución del arte anterior o posterior, al tiempo que se formulaban alternativas para el arte de su tiempo. Este contexto académico llevaría a Kaufmann a investigar sobre la transición del barroco al neoclasicismo ya desde su trabajo de doctorado y, simultáneamente, a posicionarse respecto de la arquitectura de sus contemporáneos.

\section{La analogía ética: Le Corbusier comprometido}

Kaufmann menciona por primera vez a Le Corbusier en una nota al pie del artículo "Die Stadt" (1931). ${ }^{12}$ En concreto, se remite a tres extractos de L'Architecture considerée sous le rapport de l'art, des moeurs et de la législation y destaca las similitudes entre postulados centrales de Ledoux y el libro de 1923 Vers une architecture. Estas citas, pese a no mencionarlo explícitamente, señalan una llamativa cercanía con el manifiesto lecorbusierano:

1. "Le sentiment apprécié d'un plan est à l'abri de toute domination. Il émane du sujet, il doit adapter à la nature des lieux et des besoins". ${ }^{13}$ La confianza expresada sin ambages por Le Corbusier en la capacidad generatriz de los planes combina, al igual que en esta cita de Ledoux, el carácter de autodeterminación del sujeto (que se traslada a la obra de arquitectura por medio de los procesos de abstracción) y la capacidad de estos mecanismos de ordenación de transmitir emociones: "Si ces volumes sont formels et non dégradés par des altérations intempestives, si l'ordonnance qui les groupe exprime un rythme clair, et non pas une agglomération incohérente, si les rapports des volumes et de l'espace sont faits de proportions justes, l'œil transmet au cerveau des sensations coordonnées et l'esprit en dégage des satisfactions d'un ordre élevé: c'est l'architecture". ${ }^{14}$

2. “Tout détail est inutile, je dis plus, nuisible, quand il devise les surfaces par des additions mesquines ou mensongères." " Con esta cita, Kaufmann alude a la dialéctica, con frecuencia conflictiva, que se establece en la arquitectura entre el volumen y las superficies que lo envuelven. A este tenor, el reto para Le Corbusier estriba en que estas segundas no "dévorent le volume et l'absorbent à leur profit", 16 como denuncia ser el caso en su época. En las superficies, pues, predomina el carácter utilitario (como es el caso en las edificaciones industriales) y toda adición (superflua, esto es, inútil) ha de ser entendida en este sentido como un engaño.

3. “Toutes les formes que l'on décrit d'un seul trait de compas sont avouées par le goût. Le cercle, le carré, voilà les lettres alphabétiques que les auteurs emploient dans la texture des meilleurs ouvrages. "17 Las referencias a la idea de que las formas primarias son las formas bellas abundan en Vers une architecture. Además, coincidirían Ledoux y Le Corbusier en justificarlo aduciendo la preeminencia de lo visual en el juicio sobre la arquitectura. Para Ledoux, sus proyectos "parlent aux yeux" ${ }^{, 18} \mathrm{y}$, así, para Le Corbusier en su célebre dictum: "L'architecture est le jeu savant, correct et

\footnotetext{
${ }^{12}$ Kaufmann, Emil: "Die Stadt des Architekten Ledoux”. En Kunstwissenschaftliche Forschungen, vol. 2, 1933. p. 141.

${ }^{13}$ Ledoux, Claude-Nicolas (1804): L'Architecture considerée sous le rapport de l'art, des moeurs et de la législation. París, calle Neuve d'Orléans: imprenta de H-L-Perroneau. Vol. 1, p. 65.

${ }^{14}$ Le Corbusier: Vers une architecture. $11^{\mathrm{a}}$ edición. París: Les éditions Crès et $C^{\mathrm{ia}}$, 1925. p. 35.

${ }^{15}$ Ledoux, op. cit., 1804. p. 91.

${ }^{16}$ Le Corbusier, op. cit., 1925. p. 25.

${ }^{17}$ Ledoux, op. cit., 1804. p. 135.

${ }^{18}$ Véase, por ejemplo, ibíd., pp. 52 y 115.
} 
magnifique des volumes assemblés sous la lumière. Nos yeux sont fait pour voir les formes sous la lumière; les ombres et les clairs révèlent les formes; les cubes, les cônes, les sphères, les cylindres ou les pyramides sont les grandes formes primaires que la lumière révèle bien; l'image nous en et nette et tangible, sans ambigüité." 19 Insistirán ambos, asimismo, en la capacidad para resolver los grandes problemas de la construcción moderna por medio de purismos geométricos. ${ }^{20}$

Luego, de una forma un tanto genérica, Kaufmann apunta con estas citas a tres lugares comunes de la arquitectura de Le Corbusier de entreguerras condensados en "Las tres advertencias a los señores arquitectos" de su personal manifiesto (aunque en orden inverso): el plan, la superficie y el volumen. Estas máximas aún no están explícitamente vinculadas a la autonomía de la arquitectura, aunque, como veremos en breve, ya apuntaban hacia alguna de sus características más notables. De hecho, por aquel entonces, Kaufmann todavía no había desarrollado el corpus teórico que le llevaría a ser considerado el padre putativo de la autonomía de la arquitectura. $^{21}$

Es fundamentalmente a partir de la publicación en 1933 de Von Ledoux bis Le Corbusier. Ursprung und Entwicklung der autonomen Architektur cuando queda ya irremisiblemente sellado el matrimonio entre estos dos arquitectos con la bendición del novedoso concepto de "autonomía de la arquitectura". Por aquel entonces, Kaufmann disponía no sólo ya de la versión francesa de Vers une architecture, sino que Urbanisme había sido traducida [Städtebau] y hay constancia de su adquisición del primer volumen de Oeuvres complètes. ${ }^{22}$ En este breve libro, apenas tres meses después del incendio del Reichstag, Kaufmann traza una continuidad entre los valores democráticos y republicanos de la Revolución Francesa con la arquitectura de Le Corbusier. Y, en esta línea de pensamiento, identifica a Ledoux como el precursor de un cambio de época que cristaliza heroicamente en las vanguardias arquitectónicas. La identificación del pavillonsystem en la obra de Ledoux permite a Kaufmann seguir el rastro de lo que posteriormente llamará las "configuraciones revolucionarias", pasando por la obra de Durand, Dubut, Loos, Gropius y Neutra. Esta fundación geométrico-funcional del "sistema de pabellones" en Ledoux abre la posibilidad de la supervivencia de los principios modernos más allá de los cambios de apariencia, achacados a los gustos y estilos de cada época.

Sin entrar en consideraciones relativas a la veracidad y precisión de sus análisis o, muy especialmente, de la analogía entre la arquitectura de Ledoux y aquella de Le Corbusier, es importante reseñar aquí la deliberada superficialidad con la que Kaufmann analiza la obra de Le Corbusier, tanto escrita, como construida. Así se justifica él:

Llevar a cabo una interpretación de la esencia de la arquitectura actual no puede ser objeto de un trabajo de investigación histórica. Una demostración circunstanciada del dominio de autonomía en el siglo XX parece, además, tan superflua como necesaria fue la comprobación de su existencia en el XIX. Los testimonios del modo de pensar arquitectónico de la era Le Corbusier [esto es, la era de "la arquitectura autónoma"] aparecen con tan consumada nitidez ante nuestros ojos, con una claridad tan meridiana, que hacen vanos los análisis minuciosos al respecto. ${ }^{23}$

\footnotetext{
${ }^{19}$ Le Corbusier, op. cit., 1925. p. 16.

${ }^{20}$ En la segunda advertencia: "La surface". En ibíd., pp. 25-29.

${ }^{21}$ El concepto de "autonomía de la arquitectura" apenas si había sido mencionado en un breve estudio sobre la iglesia de Chaux de Ledoux en Kaufmann, Emil: “C.N. Ledoux under der klassizistische Kirchenbau”. En Kirchenkunst, 3, 1931. p. 62.

${ }^{22}$ Véase Vidler, Anthony: Histories of the Immediate Present: Inventing Architectural Modernism, Cambridge: The MIT Press, 2008. p. 40.

${ }^{23}$ Kaufmann, Emil: De Ledoux a Le Corbusier. Origen y desarrollo de la arquitectura autónoma. Barcelona: Gustavo Gili, 1982. pp. 93-94.
} 
No es de extrañar, pues, la práctica ausencia de este presunto protagonista en la publicación. De hecho, aparece fugazmente ya terminado el texto y sin la reproducción (que contrasta con la profusión gráfica del caso de Ledoux) de ninguno de sus proyectos. Pero, más allá de la aducida evidencia del paralelismo entre Ledoux y Le Corbusier, como bien señala Vidler, diríase que su omisión en la argumentación responde a otras motivaciones. ${ }^{24}$ La teoría de la "arquitectura autónoma" de Kaufmann fue inicialmente concebida para legitimar a los arquitectos de vanguardia frente tanto al auge del neoclasicismo de parada defendido por Albert Speer, como a los críticos de la Neue Sachlichkeit (bien en su versión conservadora como Sedlmayr, bien desde el régimen estalinista). Luego, por una parte, el análisis concreto de la obra de Le Corbusier no se encontraba entre sus objetivos y de ahí que aparezca en apenas tres páginas como la cristalización de una nueva era, como el representante más autoconsciente de una incipiente revolución en la arquitectura. Y, por otra parte, al querer extrapolar su tesis al conjunto de la arquitectura amenazada por los totalitarismos, el protagonismo efectivo de Le Corbusier (más allá del meramente representativo) quedó diluido en favor de una pluralidad desde Loos y Berlage, hasta Gorpius y Neutra. Así explicitó en sus conclusiones:

la continuidad del desarrollo de la arquitectura postrevolucionaria es demostrable hasta el comienzo de nuestra propia era, que se inicia hacia 1900 con el holandés Berlage y el austriaco Adolf Loos y que puede ser caracterizada con la máxima precisión con sólo nombrar a su más conocido adalid, el líder de la joven Francia, Le Corbusier [den Führer des jungen Frankreich]. ${ }^{25}$

Más allá de estas consideraciones, como podremos comprobar en breve, la mención a Le Corbusier en su provocativo manifiesto tuvo repercusiones notables a lo largo del siglo XX en lo que respecta a su recepción. Sintéticamente, Kaufmann encuentra en Ledoux, por analogía, al liberador del régimen feudal y absolutista del barroco al incorporar en su arquitectura las concepciones morales y prácticas de la naciente burguesía francesa, como son el individualismo y el republicanismo. Es en este sentido en el que Kaufmann llega a considerar su arquitectura como "revolucionaria". Su lucha por la autonomía era la lucha de la autodeterminación contra el dictado de la necesidad externa, la razón contra la contingencia, lo sintético contra lo orgánico, lo puro contra lo corrupto. Esta debe ser considerada, en opinión de Kaufmann, la máxima aportación del periodo revolucionario. Y esta será, en definitiva, la herencia por los jóvenes arquitectos vanguardistas que él quiso enfatizar con el término "arquitectura autónoma" y con la apelación a Le Corbusier:

La semejanza de la era Ledoux con nuestro tiempo no se limita -y sea dicho esto a modo de conclusión-a los aspectos formales y temáticos. Esta similitud no nace únicamente del hecho de que, ahora como entonces, el vasto y nuevo problema de la cuestión social irrumpe con intransigencia, ávido de fuertes estímulos hacia soluciones independientes, incidiendo de manera decisiva sobre toda actividad arquitectónica. Independientemente de las nuevas exigencias de la realidad, es detectable en una y otra época un renovado idealismo, que aparece en L'Architecture de Ledoux y en los escritos de Le Corbusier, en el concepto de la Ciudad Ideal del primero y acaso en la Cité Mondial del segundo. En este idealismo, basado en un modo de entender la moralidad y el derecho completamente transformado, nos parece que estriba la verdadera $y$ última razón de la renovación arquitectónica. ${ }^{26}$

\footnotetext{
${ }^{24}$ Vidler, op. cit., 2008. pp. 37-41.

${ }^{25}$ Kaufmann, op. cit., 1982. p. 93.

${ }^{26}$ Kaufmann, op. cit., 1982. pp. 94-95.
} 
De ahí que Kaufmann critique el abuso de lugares comunes en la recepción de Le Corbusier, como serían su "fascinación por la línea recta" o el "regreso a los principios fundamentales de la esfera, el cubo y el cilindro" ${ }^{27}$ y reivindique la restauración del cambio de paradigma en la arquitectura: un arte comprometido con la sociedad, dispuesto a aliviar sus necesidades, a proporcionar nuevas perspectivas de desarrollo tanto personal como social, a mejorar, en definitiva, el estado de la cuestión.

Puesto que Le Corbusier cree tanto en este nuevo arte como podría hacerlo Ledoux, puesto que en uno y otro se percibe una íntima unión entre arte y vida por todo ello, debe mencionarse al arquitecto cuya obra supone la plena afirmación de los nuevos principios al lado de aquel otro cuyas facultades creadoras marcaron su comienzo. ${ }^{28}$

\section{Le Corbusier formalista}

Tras el Anschluss de marzo de 1938, Kaufmann tuvo que exiliarse a Estados Unidos tanto por su condición de judío como por las ideas expresadas en sus publicaciones. En su debut en 1942 en la American Society of Architectural Historians, ${ }^{29}$ en una decidida apuesta por agradar a su audiencia -nuevo entorno en el que iba a desarrollar toda su futura obra académica-, obvia toda referencia al impulso ético de su precedente teoría de la "arquitectura revolucionaria" e invierte la prioridad entre la forma y sistema en beneficio de lo que llamará "new individualism" 30 Toda mención al carácter emancipatorio de la analogía establecida entre Ledoux y Le Corbusier desaparece en beneficio de un tipo de formalismo que será explotado con profusión por sus anfitriones. Por otra parte, y en coherencia con ello, su atención prioritaria al ensalzamiento de los arquitectos de vanguardia será en lo sucesivo sintomáticamente sustituida por estudios historiográficos de la arquitectura europea del siglo XVIII y principios del XIX. ${ }^{31}$ Ciertos autores han visto en este giro una suerte de renuncia a sus primeras tesis, explícitamente orientadas hacia los valores revolucionarios franceses. Sea así o no, lo cierto es que esta versión despolitizada de su concepto de "autonomía de la arquitectura" será la que llegará con más fuerza al auditorio estadounidense, ansioso por redefinir las máximas hasta ahora heredadas del viejo continente. Serán otros, por lo tanto, los que insistan en la analogía entre Le Corbusier y las teorías sobre la "arquitectura autónoma".

Muy tempranamente, Phillip Johnson se vio atraído por la teoría de la autonomía en la arquitectura del historiador vienés y, en 1942, presentó públicamente el Von Ledoux bis Le Corbusier en la American Society of Architectural Historians en Cambridge. El individualismo, la creatividad y la originalidad defendidas por Kaufmann encajaban con su manera de entender la modernidad arquitectónica. Pero no sólo. Desde su personal

\footnotetext{
${ }^{27}$ Podría incluso interpretarse este pasaje como un ajuste de cuentas personal, en la medida en que casi contradice literalmente la primera e inmediatamente anterior alusión a Le Corbusier en "Die Stadt". En una nota al pie, dice oponerse al análisis de Stonorov y Boesiger en Le Corbusier \& P. Jeanneret de 1930.

${ }^{28}$ Kaufmann, op. cit., 1982. p. 95.

${ }^{29}$ Publicado como Kaufmann, Emil: "Claude-Nicolas Ledoux: Inaugurator of a New Architectural System”. En Journal of the American Society of Architectural Historians 3, 1943. pp. 12-20.

${ }^{30}$ Véase Mertins, Detlef: "System and Freedom. Sigfried Giedion, Emil Kaufmann, and the Constitution of Architectural Modernity”. En Somol, Robert E. (Ed.): Modernity and Ideology: Positioning an Avant-Garde in America. Nueva York: The Monacelli Press, 1997. pp. 212-231.

${ }^{31}$ Estos estudios se plasmarán a lo largo de su carrera en sus dos publicaciones de mayor impacto: Three Revolutionary Architects: Boullée, Ledoux, Lequeu (1952) y su magnus opus póstuma Architecture in the Age of Reason: Baroque and PostBaroque in England, Italy, and France (1955).
} 
interpretación de Kaufmann, este postulado sintonizaba con su trayectoria profesional y, en especial, con su empeño de centrar toda discusión sobre la arquitectura en una suerte de indeterminación ideológica.

Desde la exposición "Rejected Architects" de 1931, Johnson había espoleado una controversia en Estados Unidos de América que no tardaría en llegar a su momento culminante. Como atestiguan diferentes críticos de la época, la exposición se entendió como una intromisión de outsiders en la arquitectura y dinámicas culturales estadounidenses que los desviaban de sus objetivos que remitían a una tradición de profunda raigambre social. Douglas Haskell, por ejemplo, reivindicó el compromiso nacionalista y la tecnocracia local frente a esos "postulados extranjeros". De forma más explicita, Catherin Bauer plasmaba su preocupación al constatar que apenas se percibían en la exposición compromiso con la sociedad, de no ser por los representantes locales Alfred Claus y George Daub. ${ }^{32}$ Así pues, desde entonces, en un amplio sector de la crítica arquitectónica de esa época se vinculó el protagonismo de Le Corbusier a la figura del arquitecto ajeno a las demandas de la sociedad, y a Kaufmann y Johnson, sus adalides, como su embajador. La tan aclamada International Style Exhibition de 1932 del Museum of Modern Art de Nueva York, organizada y catalogada por Henry-Russel Hitchcock, Alfred Barr y Phillip Johnson, supuso la culminación de esta agitada polémica, llevando a sus autores a instalarse en un radicalismo formal inédito hasta entonces. Como Johnson afirmará años más tarde: "the functionalist part and the Marxian part [de la arquitectura moderna] left me cold, because to me architecture is a pure art, and it's art that interests $m e " .{ }^{33}$ En detrimento del compromiso con lo social que enarbolaron explícitamente las vanguardias europeas, la "autonomía de la arquitectura" que Kaufmann defendió para justificar la obra de, entre otros, Le Corbusier se interpretó en el entorno de Johnson como una revolución, sí, pero exclusivamente lingüística. Con su acostumbrada vehemencia, recordaba así su fascinación por la arquitectura europea de entreguerras: "In fact, we did not have the slightest idea what the avant-garde was. [...] At the Museum of Modern Art, we were ignorant of the political dimension of the art; for us it was revolutionary, but only aesthetically". ${ }^{34}$ Su pretendida falta de compromiso apela a una falta de interés por las implicaciones sociales del hecho arquitectónico. Lo que realmente interesaba a Johnson era el desarrollo de un lenguaje arquitectónico propio, "puro", en sus propios términos, que vinculaba a la tradición desempolvada por Kaufmann. ${ }^{35}$

Con la publicación del catálogo International Style: Architecture since 1922, quedó meridianamente claro cuáles eran los puntos a rescatar de las vanguardias arquitectónicas. Destacaron tres principios básicos que venían avalados por las obras expuestas de Le Corbusier. ${ }^{36}$ En primer lugar, haciendo uso de los progresos tecnológicos, los edificios modernos podían pasar a ser "mere planes surrounding a volume", a diferencia de las construcciones clásicas, donde los muros servían tanto como estructura como divisoria espacial. Con la incorporación del acero y del hormigón armado a la arquitectura se permitió la separación entre la fachada y la planta. En este sentido, el efecto de masa de la arquitectura tradicional se sustituyó por "plane surfaces bounding a volume". ${ }^{37}$ En segundo lugar, la regularidad atendía simultáneamente a los requerimientos de tipo económico e industrial y al principio impuesto de fragmentación por el cual cada una de las funciones han de ser

\footnotetext{
${ }^{32}$ Véase Baco, Mardges: Le Corbusier in America: Travels in the Land of the Timid. Cambridge: The MIT Press, 2001. p. 22.

${ }^{33}$ Lewis, Hilary y O’Connor, John: Philip Johnson: the architect in his own words. Nueva York: Rizzoli, 1994. p. 174.

${ }^{34}$ Somol, op. cit., 1997. p. 42.

${ }^{35}$ En 1950, publicará un artículo en The Architectural Review donde argumenta que la forma de su Glass House es heredera de la pureza geométrica de Ledoux. Véase Schulze, Franz: Philip Johnson. Life and Work. Nueva York: Knopf, 1994. pp. $157-158,194-196$ у 216.

${ }^{36}$ Como muestra del reconocimiento al protagonismo de Le Corbusier en "Modern Architecture: International Exhibition", mostraron fotografías y dibujos de sus casas de la Weissenhof Siedlung de Stuttgart, el pabellón suizo en la Cité Universitaire de París y varias villas. Pero, por encima de cualquier otra, tanto en la exposición (y su icónica maqueta) como en el catálogo (ocupando la portada como única representación gráfica) la indiscutible protagonista fue la Villa Savoye.

${ }^{37}$ Somol, op. cit., 1997. p. 56.
} 
identificables por sí mismas, sin requerir de la mediación de otras para justificarse. Así, por ejemplo, las ventanas, que anteriormente tenían valor de contraste con la masa del muro, pasaron a ser superficies acristaladas de grandes dimensiones. ${ }^{38}$ Por último, y coherentemente con este principio de fragmentación, los detalles constructivos en la modernidad así interpretada por Johnson (y Hitchcock) adquieren valor arquitectónico: las barandas, las ventanas, los parapetos dejan de estar afectados por el tradicional intrusismo de otras disciplinas como la escultura o la pintura y, en definitiva, dejan de estar sobreimpuestos al volumen. ${ }^{39}$

La "architecture as Volume" en lugar de la arquitectura entendida como masa, la "regularity" y "the avoidance of applied decoration" o la no intromisión de otras disciplinas recuerdan los primeros postulados de Kaufmann (luego tan duramente criticados por él mismo) recogidos de Vers une architecture en 1931. En este sentido, puede considerarse International Style: Architecture since 1922 como el acta fundacional de una nueva manera de entender la autonomía de la arquitectura, en la que sus paradigmas quedan reducidos a operaciones de tipo formal desprovistas ya de toda carga ética.

El impacto de esta exposición y su catálogo es público y notorio, de suerte que este giro en la recepción de Le Corbusier y su explícito vínculo con las teorías de la arquitectura autónoma impregnará notablemente la recepción anglosajona de la obra de Le Corbusier respecto de su relación con la autonomía en las décadas siguientes. Como muestra de ello, autores como Hilberseimer o Gandelsonas (en diversas publicaciones de Oppositions) seguirán insistiendo en las cualidades autorreferenciales de la arquitectura de Le Corbusier, enfatizando el purismo de sus operaciones formales. Es significativo a este respecto cómo se erigirá la Maison Dom-ino a paradigma de sus postulados. Así lo defendió Gandelsonas en 1976:

But are any or all these variations anything more than geometry? And even in terms of their use as floor levels and the necessity to enclose them so as to provide shelter, are they anything more than a set of geometric relationships plus this use, which together in some way approximate what we have always thought architecture to be? And if we answer in the affirmative that they do constitute architecture, then do all such variations of these elements when combined with their uses constitute architecture? And if it immediately appears clear that not all of the examples qualify, then how do we begin to distinguish between those that do not?. ${ }^{40}$

Por su parte, Hilberseimer reunió en Contemporary Architecture (1964) el constructivismo ruso, el neoplasticismo holandés y el "lecorbusierano purismo" bajo el concepto de "arquitectura autónoma".

\section{Le Corbusier y el fin de los grandes relatos}

Si esto es así entre aquellos que confiaron -incluso durante los años 70- en la aproximación formal heredada de Johnson, cabe ahora recordar los movimientos contestatarios que operaron a lo largo de esas décadas y, muy especialmente, a partir del 68.

Por aquel entonces, el malestar en la disciplina era patente a un lado y otro del Atlántico. Desde muy diversos posicionamientos predominaba la crítica a la reinterpretación dogmática de la modernidad arquitectónica, monopolio que había degenerado en un "estilo desenraizado, intelectualizado y superficial”. En este contexto, al igual que el "funcionalismo", la idea de una arquitectura intelectual, elitista, universal o sobre-impuesta, la

\footnotetext{
${ }^{38}$ Ibíd., p. 73.

${ }^{39}$ Ibíd., p. 85.

${ }^{40}$ Gandelsonas, Mario: “Aspects of Modernism: Maison Dom-ino and the Self-Referential Sign”. En Oppositions, vols. 1516, The Institute for Architecture \& Urban Studies, 1976. pp. 188-199.
} 
"autonomía de la arquitectura" fue objeto de duras críticas. La estrategia de Johnson no garantizó a ojos de la nueva generación -salvo contadas excepciones ${ }^{-41}$ una alternativa al imparable auge de las lógicas postindustriales y, por otra parte, había consolidado un problemático alejamiento de la arquitectura respecto de las necesidades sociales.

La radicalización de las posiciones llevó al cultivo de los extremos, según los cuales la arquitectura ha de anteponer su autonomía como disciplina a su integración en la sociedad; y el opuesto, según el cual la arquitectura antes que nada es social y, si quiere llevar a cabo su misión crítica, ha de desprenderse del aislamiento al que le llevó el discurso autónomo formal. En cualquier caso, tanto los defensores de la posible recuperación del proyecto de autonomía en la arquitectura como sus detractores, partieron de la necesidad de incorporar un giro social a la arquitectura. Así, para unos, el compromiso social suponía la recuperación del proyecto de vanguardia de formación de estilos de vida: el objetivo de orientar la arquitectura hacia la reforma de la sociedad, de responder a sus necesidades. Autores como Summerson o Banham abrazaron, pues, el carácter mesiánico del movimiento moderno (en crítica directa a la recepción de Johnson y el "Internationnal Style"), ${ }^{42}$ es decir, la necesidad de orientar la arquitectura según fines ajenos a ella, hacerse social a costa de perder su singularidad, renunciando por supuesto a su autonomía. Para otros, en cambio, la implementación de la crítica en la arquitectura suponía por fuerza la recuperación del proyecto ilustrado de autonomía más propiamente kantiano. ${ }^{43}$ En última instancia, este posicionamiento veía en las vanguardias arquitectónicas el riesgo constatado en la posguerra- de confundirse con aquello contra lo cual se disponían a luchar. La no delimitación clara de las fronteras de la arquitectura respecto de otras prácticas y de otros factores sociales podía llevar a una complicidad con los responsables de aquello que se quería transformar. En consecuencia, esta tendencia de la década de los 70 -con el explícito apelativo de autonomy project- tuvo que enfrentarse en primer lugar a la delimitación de dichas fronteras con el fin de evitar su disolución en lo social. Así lo sintetizaba K. Michael Hays en el monográfico de Perspecta veinte años después:

The various researches into architecture's autonomy can now be understood in their historical trajectory as nothing quite so much as attempts to recode, to reterritorialize, to reinvent the boundaries and specificities that delimit the discipline. ${ }^{44}$

Considerar la arquitectura simultáneamente como un arte autónomo y como parte de la sociedad (en la terminología corriente en estas discusiones, como "formal product" y como "cultural act") fue la estrategia ampliamente consensuada para superar el aislamiento en el que había caído en la posmodernidad y poder simultáneamente recuperar su capacidad transformadora de la sociedad. Desde diferentes presupuestos, y a modo de mera contextualización, autores como K. Michael Hays, Diana Agrest, Stanford Anderson, Peter Eisenman, Alan Colquhoun o Anthony Vidler defendieron cómo al ser parte de la cultura y servirle, la arquitectura detenta

\footnotetext{
${ }^{41}$ Obviando aquellos que, aún siendo activos a finales de los 60 y las décadas siguientes, pertenecían a la generación anterior, sólo la "escuela formalista" siguió defendiendo la autonomía de la arquitectura desde una gramática rígida de las formas autorreferentes (una aproximación cercana a la de Rossi, por otra parte).

${ }^{42}$ Banham, Reyner: “Actual Monuments”. 1988. En Banham, Mary y otros (Ed.). A Critic Writes Selected Essays by Reyner Banham, Los Angeles: University of California Press, 1999. p. 285.

${ }^{43}$ Véase Hays, K. Michael: "Prolegomenon for a Study Linking the Advanced Architecture of the Present to that of the 1970s through Ideologies of Media, the Experience of Cities in Transition, and the Ongoing effects of Reification". En Perspecta: the Yale Architectural Journal, vol. 32, Cambridge: Yale University, 2001. pp. 100-107.

${ }^{44}$ Hays, K. Michael: "Twenty Projects at the Boundaries of the Architectural Discipline Examined in Relation to the Historial and Contemporary Debates over Autonomy". En Perspecta: the Yale Architectural Journal, vol. 33: Minning Autonomy, Connecticut: Yale University, 2002. p. 55.
} 
un potencial de transformación de lo social, sin por ello tener que renunciar a su autonomía. Las diferencias entre tan variados referentes son, por supuesto, notables en lo que respecta a la elaboración de un programa alternativo para la autonomía de la arquitectura. No obstante, adentrarnos en este complejo panorama excede el propósito de este texto. Por tanto, me limitaré en lo que sigue a apuntar someramente dos de las principales corrientes que siguieron enarbolando la obra de Le Corbusier - aún con llamativas diferencias- en favor de una rehabilitación de los paradigmas autonomicistas, sin llegar a matizar como cabría en otro contexto qué se entiende en cada caso por "autonomía de la arquitectura".

Desde una perspectiva escorada del lado del "formal product", para Alan Colquhoun la modernidad supone entender la forma en la arquitectura como indisoluble de una "idea" que emerge desde sus valores propios:

Composition therefore was able to stand for an aesthetic of immanence in which art became an independent kind of knowledge of the world and was no longer, as it had been both in the medieval and the classical traditions, the means by which certain "truths" or concepts were given rhetorical clothing. ${ }^{45}$

La forma arquitectónica, pues, albergaría por derecho propio sus valores estéticos, independientes de toda realidad externa a ella misma. De ahí que vea en Le Corbusier un privilegiado representante de este nuevo potencial, en la medida en que formuló las innovaciones tecnológicas, por ejemplo, con medios inherentes a la arquitectura. Su conocida fórmula de "la casa es una máquina de vivir" no sólo apuntaría en este sentido a las posibilidades técnicas, sino que enfatiza sus ramificaciones estéticas:

It was not to annex architecture to a branch of empirical science, but to use the machine as a model for a work of art whose form and structure were determined by laws internal to itself. The laws which applied to technology were different from those which applied to architecture, the first being directed to the solution of practical problems, the second to the creation of states of mind. In both cases, however, the desired results could only be obtained by understanding the laws which controlled their production. From this point of view Le Corbusier's famous statement can be interpreted as a metaphor for an aesthetic theory... ${ }^{46}$

Sería legítimo, en este sentido, incorporar a la disciplina arquitectónica elementos ajenos, tales como la técnica o los requerimientos funcionales, siempre y cuando se haga en clave arquitectónica. Colquhoun insiste así en la unificación en el seno de la arquitectura de la ciencia y el arte, y como muestra de ello se refiere a la comparación de Le Corbusier entre el Partenón y un automóvil moderno en las páginas de Vers une architecture: estos dos elementos, en principio ajenos el uno al otro, revelan propiedades comunes como el orden o la invariabilidad, propiedades que forman parte de los valores estéticos de la arquitectura.

En segundo lugar, desde una perspectiva materialista y, por tanto, que insiste en el carácter de "cultural act", la arquitectura no debería ser evaluada como un "passive agent of culture" que simplemente refleja las fuerzas sociales e históricas dominantes, sino como una entidad activa que tiene un lugar en la cultura "as an architectural intention with ascertainable political and intellectual consequences". ${ }^{47}$ Luego, la arquitectura

\footnotetext{
${ }^{45}$ Colquhoun, Alan: Modernity and the Classical Tradition: Architectural Essays, 1980-1987. Cambridge: MIT Press, 1989. p. 34.

${ }^{46}$ Colquhoun, Alan: "The Significance of Le Corbusier". En Brooks, H. Allen (Ed.): Le Corbusier: the Garland Essays. Nueva York: Garland, 1987.

${ }^{47}$ Hays, K. Michael: "Critical Architecture: Between Culture and Form”. En Perspecta: the Yale Architectural Journal, vol.
} 
constaría de diferentes niveles, alguno de los cuales tiene propiedades interiores y otros que requieren la comunicación con la realidad exterior. Ambos niveles se relacionan entre sí, dando lugar a una arquitectura espacial y temporalmente localizada: la "social formation". Dado que sus diferentes niveles se comunican con las realidades sociales y culturales, la arquitectura no puede ser totalmente independiente de lo social:

At a different level of autonomy thesis there appears a key concept from Louis Althusser, that of the "semi-autonomy" of "levels" or "instances" within an ideological field -the economic, political, juridical, cultural, aesthetic realms (and so on). The autonomy of each disciplinary level allows the development and advance of that discipline's particular techniques. But each level also feels pressure from all the others and exerts influence on all the others. What results is a set of insides and outsides that are reciprocally constituted and related by way of their ultimate structural difference and distance from one another rather than their identity, all held together by the "structural totality" of a social formation. ${ }^{48}$

Esta línea de pensamiento, tan abiertamente althusseriana, fue recogida con mucha más literalidad por Stanford Anderson. En tanto que disciplina, la arquitectura ha de enfrentarse al hecho de que no puede liberarse totalmente de la función (como exterioridad) y, por otra parte, no puede operar de forma totalmente funcional: "what we have is a spectrum that has to be analyzed for the attitudes of the architects, for theresponse that we expect relative to a particular kind of problem that the building is addressing". Para salvar esta dificultad, Anderson propone la distinción entre "discipline" y "profession" en la arquitectura. La primera debería ser considerada como un "collective body of knowledge that is unique to architecture and which, though it grows over time, is not delimited in time or space”. La profesión, en cambio, estaría vinculada a las condiciones reales y temporales de la arquitectura. ${ }^{49}$ La disciplina de la arquitectura gozaría, pues, de un carácter temporal y espacialmente indefinido. Posteriormente, con el concepto de "quasi-autonomy", Anderson evaluará la autonomía de la arquitectura como un rango de posibilidades que pueden revelarse de diferentes formas autónomas o no-, como resultado de la intención de los arquitectos combinada con las circunstancias sociales y culturales que el edificio tiene que satisfacer, diluyendo por tanto el intento metódico de delimitar las fronteras disciplinares. En este sentido, Anderson da especial énfasis en "Quasi-Autonomy in Architecture" a la capacidad de cambio de estilos de vida. Referido al Carpenter Center de Le Corbusier:

First, he [Le Corbusier] made the building itself an active participant in the problem situation rather than a retiring, effortless framework. Secondly, the visitor and Harvard are forced to recognize that illiteracy about art is not a matter of vision alone. In this building art is not a spectator sport; all of one's senses and the whole of one's perception are engaged. One feels that the Carpenter Center is a world, a context, a problem, and we have the happy opportunity to form ourselves against it. ${ }^{50}$

Desde su tesis, la arquitectura es parte de la cultura, no una parte independiente como quiso entender Johnson, sino dependiente de ella, insertada en ella. Reivindica el valor de compromiso con lo social del Carpenter Center, la capacidad transformadora de lo social de la arquitectura, en la medida en que participa de los problemas que

21, Cambridge: Yale University, 1984. p. 20.

${ }^{48}$ Hays, op. cit., 2001, pp. 100-107.

49 Anderson, Stanford: "Types and Conventions in Time: Toward a History for the Duration and Change of Artifacts". En Perspecta: The Yale Architectural Journal, vol. 18, Cambridge: Yale University, 1982. p. 115.

50 Anderson, Stanford: "Quasi-Autonomy in Architecture: The Search for an 'In-between'". En Perspecta: the Yale Architectural Journal, vol. 33: Minning Autonomy, Connecticut: Yale University, 2002. pp. 30-37. 
se dan lugar en su localización. En este sentido, es una exigencia para la arquitectura, defiende Anderson, no retraerse, no quedar al margen de lo que le convoca. Es más, insiste, la arquitectura es propiamente "fundadora de mundo". En su intervención, el arquitecto es capaz de traer el arte a la vida, de hacerlo presente. De suerte que el edificio, el Carpenter Center en este caso, forma a su vez parte de ese mundo en el que la arquitectura, el arquitecto y el espectador han de tomar partido, participando simultáneamente del problema y la solución. Esta posición de Anderson sintetizada en el explícito "Quasi-Autonomy of Architecture" comparte el carácter pragmático y moderado -si lo comparamos con las dos precedentes- que abundó durante estas décadas (como Solà-Morales y su “Arquitectura débil” o Kenneth Frampton y la idea del "Critical Regionalism").

Aunque el autonomy project no reclamaba un papel utópico, como fue el caso en la arquitectura de vanguardia, la exigencia de autonomía de la arquitectura en los 70 no pretendió únicamente redefinir la disciplina sino también ganar resonancia social. En otras palabras, tras de la explícita intención por parte de la arquitectura en la década de 1970 de reinventar su territorio, los límites de su especificidad como disciplina, aflora la redeterminación de su función cultural. Los arquitectos del autonomy project abandonaron el papel heroico de la vanguardia arquitectónica (entendida como heteronomía) y trataron de recuperar la autonomía de la arquitectura renunciando a la pretensión de transformar la vida, así como cualquier responsabilidad sobre el destino del mundo. El proyecto era un decidido intento de apartar a la arquitectura de la ideología dominante y de resistencia frente al status quo, así como un empuje para volver a alcanzar un lugar en la sociedad y recuperar la labor crítica de la arquitectura moderna. No obstante, la autonomía así entendida llevó a la fragmentación de la arquitectura: internamente supuso una exigencia de descifrado esencialista y de recodificación, y externamente un dilema respecto de su terreno de intervención en otros ámbitos sociales -bajo la prohibición de una propuesta utópica y con la exigencia de una orientación social basada en la necesidad de reestructurar el presente-. En definitiva, esta fragmentación llevó a posicionamientos o bien directamente contrarios a la autonomía sensu stricto (como Anderson y su "quasi-autonomy") o intentos de recuperar una unidad perdida por medio de las teorías estructuralistas o lingüísticas (como es el caso de Colquhoun).

\section{Conclusiones}

Más allá de exponer los usos interesados de la obra tanto escrita como construida de Le Corbusier para justificar su vínculo con la autonomía de la arquitectura, el objetivo de este texto ha sido defender su centralidad en estas discusiones.

En la irrupción de la problemática asunción teórica del postulado autonomicista, Kaufmann defendió la obra de Le Corbusier como un exponente ejemplarizante del intrínseco movimiento emancipador que cabe esperar de la arquitectura en tanto que arte comprometido con la sociedad. Vio en el Mundaneum la cristalización de este postulado. Por su parte, el relevo abanderado por Johnson redujo la cuestión a una pregunta por la "forma" arquitectónica. La autonomía se entendió así como un problema de lenguaje arquitectónico, un proyecto de desarraigo y de abstracción formal. En este sentido, obras como la Villa Saboye o la Maison Dom-ino fueron erigidas como sus paradigmas. En el tercer periodo analizado, los intentos de rehabilitación de la autonomía de la arquitectura se vieron en mayor o menor medida enfrentados a la imposibilidad del proyecto y los más resolvieron tal aporía con la negación de uno de los polos: o bien se caía del lado de una autonomía restrictiva de la arquitectura, o bien se intentaba rescatar una concepción esencialista de la arquitectura. $\mathrm{O}$, en otras palabras, durante el tercer periodo no se introdujeron propiamente nuevos elementos a la discusión, sino que se entendió que la salida a los fracasos anteriores estribaba en una combinación de ambos polos. En este caso, por ejemplo, Anderson encontró en el Carpenter Center una muestra de ello. 
A la vista del significado fundacional de la autonomía, no sorprende la ductilidad de los posicionamientos, pese a que se remitan en principio a una misma tradición. Todas las interpretaciones responden a la duplicidad del significado de autonomía detectado ya en Kant. Así, tanto el "Le Corbusier comprometido" como el "Le Corbusier formalista" atienden, por sorprendente que pueda resultar, a un mismo paradigma. En efecto, de esta inestable relación entre Le Corbusier y la autonomía de la arquitectura se desprende una paradoja difícilmente salvable: o bien se suscribía la tesis formalista según la cual el compromiso con lo social se establece "despolitizando" la arquitectura, o bien se recuperaba la naturaleza social de la arquitectura, reivindicando su capacidad de transformación de la sociedad. La caída de los grandes relatos que implementó el tercer periodo no es otro que un intento de no renunciar a ninguno de los extremos.

En un sentido amplio, visto como síntoma, diríase que la autonomía apela a la necesidad de establecer un principio a priori para la arquitectura una vez hecho experiencia de la quiebra de sus fundamentos: con la perdida su razón de ser espontánea provocada por la Ilustración y sus procesos de racionalización, la arquitectura pierde su legitimidad esencial. Es llamativo en este sentido cómo las discusiones en torno a la autonomía de la arquitectura han coincidido con momentos de crisis en la disciplina que exigían su redefinición y, aun más, un posicionamiento respecto de la deriva moderna en la sociedad. Frente a la amenaza de los totalitarismos, Kaufmann inauguró el debate en 1933 con su Von Ledoux bis Le Corbusier; tras la Segunda Guerra Mundial y la crisis del modelo de vanguardia, Johnson apeló a la autonomía para refundar los códigos formales y lingüísticos de la arquitectura de posguerra; con el agotamiento de la modernidad, a lo largo de los sesenta y setenta, la autonomía tuvo que enfrentarse simultáneamente a la amenaza del populismo y a la del elitismo.

Como señala Moneo en el prólogo a la edición española de Architecture in the Age of Reason de 1974, al margen del análisis concreto que presentó Kaufmann de la arquitectura "revolucionaria", cabe reconocerle el mérito de haber planteado la hipótesis de continuidad entre la Ilustración y la arquitectura de vanguardia. Así, su acierto más destacado quizás fue el de entender la modernidad en la arquitectura como un proyecto social y la autonomía en el sentido kantiano del término como estrategia de relación entre lo social y lo formal: haciéndose autónoma, esto es, atendiendo exclusivamente a las reglas que le son propias, la arquitectura se hace hecho social. Y, en esta línea de reflexión, bien podemos defender ahora que, en estas tentativas que se prolongaron a lo largo del siglo veinte, Le Corbusier ocupa un papel central. Su obra ejemplifica, en definitiva, la modernidad arquitectónica entendida como una ineludible y paradójica dialéctica entre la forma y su función social, enlazando así con una de sus provocaciones más acertada: "Architecture ou révolution, on peut éviter la révolution?".

\section{Bibliografía}

Adorno, Theodor W.: Teoría estética. Obra completa $n^{\circ} 7$. Rolf Tiedemann (Ed.), Jorge Navarro Pérez (Trad.), Madrid: Akal, 2004

Anderson, Stanford: "Types and Conventions in Time: Toward a History for the Duration and Change of Artifacts". En Perspecta: The Yale Architectural Journal, vol. 18, Cambridge: Yale University, 1982

Baco, Mardges: Le Corbusier in America: Travels in the Land of the Timid. Cambridge: The MIT Press, 2001

Brooks, H. Allen (Ed.): Le Corbusier: the Garland Essays. Nueva York: Garland, 1987

Colquhoun, Alan: Modernity and the Classical Tradition: Architectural Essays, 1980-1987. Cambridge: MIT Press, 1989

Hays, K. Michael: “Critical Architecture: Between Culture and Form”. En Perspecta: the Yale Architectural Journal, vol. 21, Cambridge: Yale University, 1984 
Hitchcock, Henry Russell y Johnson, Philip: The International Style: Architecture Since 1922. W. W. Norton, Incorporated, 1932

Kant, Immanuel: Fundamentación de la metafísica de las costumbres. Madrid: Espasa Calpe, 1990

Kant, Immanuel: Crítica del Juicio. Manuel García Morente (Trad.), Madrid: Tecnos, 2007

Le Corbusier: Vers une architecture. $11^{\text {a }}$ edición. París: Les éditions Crès et $C^{\text {ia }}, 1925$

Ledoux, Claude-Nicolas: L'Architecture considerée sous le rapport de l'art, des moeurs et de la législation. Vol. 1. París, calle Neuve d'Orléans: imprenta de H-L-Perroneau, 1804

Perspecta: the Yale Architectural Journal, vol. 33: Minning Autonomy

Somol, Robert E. (Ed.): Modernity and Ideology: Positioning an Avant-Garde in America. Nueva York: The Monacelli Press, 1997

Vidler, Anthony: Histories of the Immediate Present: Inventing Architectural Modernism, Cambridge: The MIT Press, 2008 